\title{
ON THE TRANSCENDENCE OF SOME INFINITE SERIES
}

\author{
JAROSLAV HANČL* AND JAN ŠTĚPNIČKA \\ Department of Mathematics and Institute for Research and Applications of Fuzzy Modeling, University of \\ Ostrava, 30. dubna 22, 70103 Ostrava 1, Czech Republic \\ e-mail: hancl@osu.cz, janstepnicka@centrum.cz
}

(Received 23 June, 2006; accepted 31 July, 2007)

\begin{abstract}
The paper deals with a criterion for the sum of a special series to be a transcendental number. The result does not make use of divisibility properties or any kind of equation and depends only on the random oscillation of convergence.
\end{abstract}

2000 Mathematics Subject Classification. 11J81.

1. Introduction. Erdös [2] proved that if $\left\{a_{n}\right\}_{n=1}^{\infty}$ is an increasing sequence of positive integers such that $\lim _{n \rightarrow \infty} a_{n}^{\frac{1}{2^{n}}}=\infty$ then the number $S=\sum_{n=1}^{\infty} \frac{1}{a_{n}}$ is irrational. In [4] it is shown that if $\lim _{n \rightarrow \infty} \log _{3} \log _{2} a_{n}>1$ and $a_{n} \in \mathbb{N}$ for all $n \in \mathbb{N}$ then $S$ is a transcendental number. Many other criteria for $S$ to be transcendental can be found in [1], [5], [6] or [8] but divisibility properties or fullfilling special equations are necessary. It seems to be the case that in general it is not easy to decide when $S$ is a transcendental number if $\limsup _{n \rightarrow \infty} \log _{3} \log _{2} a_{n}<1$ holds and divisibility properties or fullfilling certain equations are not required. In this paper we give conditions on sequences $\left\{a_{n}\right\}_{n=1}^{\infty}$ with $\lim \sup _{n \rightarrow \infty} \log _{3} \log _{2} a_{n}<1$ such that $S$ is transcendental. We prove the following.

THEOREM 1.1. Let $\left\{a_{n}\right\}_{n=1}^{\infty}$ be an eventually non-decreasing sequence of positive integers such that $a_{n}>2^{n}$ for every sufficiently large $n$. Suppose that $a_{n}<2^{3^{\frac{1}{4} n}}$ and that $a_{2 n}>2^{3^{\frac{3}{4}(2 n)}}$ for infinitely many $n$. Then the number $\sum_{n=1}^{\infty} \frac{1}{a_{n}}$ is transcendental.

EXAMPLE 1.1. Let $a_{1}=a_{2}=1$. For every $s=0,1,2,3, \ldots$ set

$$
a_{n}= \begin{cases}2^{\left[3^{\frac{1}{4}\left(5 n-42^{3^{s}}\right)}\right]}+3 & \text { if } 2^{3^{s}}<n \leq 2.2^{3^{s}} \\ 2^{\left[3^{\frac{6}{4^{3^{s}}}}\right]}+2^{\left[3^{\frac{1}{4^{n}}}\right]}+3 & \text { if } 2.2^{3^{s}}<n \leq 2^{3^{s+1}}\end{cases}
$$

Then the number $\sum_{n=1}^{\infty} \frac{1}{a_{n}}$ is transcendental.

It is unclear to the authors if there exists a sequence $\left\{a_{n}\right\}_{n=1}^{\infty}$ of positive integers such that $\sum_{n=1}^{\infty} \frac{1}{a_{n}}$ is an algebraic number and $a_{n}>2^{\left(\frac{5}{2}\right)^{n}}$ for all $n \in \mathbb{N}$.

2. Main results. In the sequel, for a real number $x$ we use $[x]$ to denote the greatest integer less than or equal $x$. Theorem 1.1 is an immediate consequence of the following theorem.

*Supported by the grants no. 201/07/0191 and MSM6198898701 
THEOREM 2.1. Let $\alpha, \beta, \gamma$ and $v$ be real numbers with $0<\beta<\alpha<\log _{2} 3,0 \leq v<$ 1 and $\gamma>0$. Assume that $\left\{a_{n}\right\}_{n=1}^{\infty}$ and $\left\{b_{n}\right\}_{n=1}^{\infty}$ are sequences of positive integers with $\left\{a_{n}\right\}_{n=1}^{\infty}$ eventually non-decreasing such that for every sufficiently large $n$

$$
b_{n}<a_{n}^{v} \log _{2}^{\gamma} a_{n}
$$

and

$$
a_{n}>2^{n} \text {. }
$$

Suppose that there exists a positive real number $k$ with

$$
k<\frac{(\alpha-\beta)}{\log _{2}\left(\frac{2}{1-\nu}+1\right)-\alpha}
$$

such that for infinitely many $n$

$$
a_{n}<2^{2^{\beta n}}
$$

and

$$
a_{n+[k . n]}>2^{2^{\alpha(n+[k . n])}} .
$$

Then the number $\sum_{n=1}^{\infty} \frac{b_{n}}{a_{n}}$ is transcendental.

As an immediate consequence of Theorem 2.1 we obtain the following corollary.

COROLlary 2.1. Let $\alpha, \beta$ and $\gamma$ be real numbers with $0<\beta<\alpha<1$ and $\gamma>0$. Assume that $\left\{a_{n}\right\}_{n=1}^{\infty}$ and $\left\{b_{n}\right\}_{n=1}^{\infty}$ are sequences of positive integers with $\left\{a_{n}\right\}_{n=1}^{\infty}$ eventually non-decreasing such that for every sufficiently large $n$

$$
b_{n}<\log _{2}^{\gamma} a_{n}
$$

and

$$
a_{n}>2^{n} .
$$

Suppose that there exists positive real number $k$ with

$$
k<\frac{(\alpha-\beta)}{1-\alpha}
$$

such that for infinitely many $n$

$$
a_{n}<2^{3^{\beta n}}
$$

and

$$
a_{n+[k . n]}>2^{3^{\alpha(n+[k . n])}} .
$$

Then the number $\sum_{n=1}^{\infty} \frac{b_{n}}{a_{n}}$ is transcendental.

REMARK 2.1. Let the sequences $\left\{a_{n}\right\}_{n=1}^{\infty}$ and $\left\{b_{n}\right\}_{n=1}^{\infty}$ satisfy all conditions (1)-(5). Then Theorem 2.1 implies that the number $\sum_{n=1}^{\infty} \frac{b_{n}}{a_{n}}$ is transcendental. If in addition 
there exists a fixed $\epsilon>0$ such that

$$
a_{n}<2^{(2-\epsilon)^{n}}
$$

holds for all sufficiently large $n$ then there exists a sequence $\left\{c_{n}\right\}_{n=1}^{\infty}$ of positive integers such that $\sum_{n=1}^{\infty} \frac{b_{n}}{c_{n} a_{n}}$ is a rational number. For more information see [3].

3. Proof. Proof. (of Theorem 2.1) Let $N$ be a sufficiently large positive integer satisfying (4) and (5). Assume that $\delta$ is a sufficiently small positive real number.

Let us define the finite sequence $\left\{c_{t}\right\}_{t=N}^{N+[k . N]}$ by

$$
c_{t}= \begin{cases}a_{t}^{t}, & \text { if } t=N \\ \frac{1}{a_{t}^{\left(\frac{2}{1-\nu}+1+\delta\right)^{t-N}}}, & \text { if } t=N+1, N+2, \ldots, N+[k \cdot N] .\end{cases}
$$

Set

$$
c_{T}=\max _{t=N, N+1, \ldots, N+[k \cdot N]} c_{t} .
$$

If $c_{T}=c_{N}$ then from (4) and (5) we obtain

$$
2^{N 2^{\beta N}}>a_{N}^{N}=c_{N} \geq c_{N+[k \cdot N]}=a_{N+[k \cdot N]}^{\frac{1}{\left(\frac{2}{1-v}+1+\delta\right)^{[k . N]}}}>2^{\frac{2^{\alpha(N+[k . N])}}{\left.\left(\frac{2}{1-\nu}+1+\delta\right)^{k} \cdot N\right]}}=2^{2^{\alpha(N+[k . N])-[k \cdot N] \log _{2}\left(\frac{2}{1-\nu}+1+\delta\right)}} .
$$

Applying $\log _{2}$ twice to the above inequality we get

$$
\log _{2} N+\beta N>\alpha(N+[k \cdot N])-[k \cdot N] \log _{2}\left(\frac{2}{1-v}+1+\delta\right)
$$

and this is a contradiction with (3).

Therefore $c_{T} \neq c_{N}$ and thus

$$
c_{T} \geq \max _{j=N, N+1, \ldots, T-1} c_{j}
$$

From this and from the fact that the sequence $\left\{a_{n}\right\}_{n=1}^{\infty}$ is eventually non-decreasing we obtain that

$$
a_{T} \geq\left(\max _{j=N, N+1, \ldots, T-1} c_{j}\right)^{\left(\frac{2}{1-\nu}+1+\delta\right)^{T-N}}>\prod_{i=N}^{T-1}\left(\max _{j=N, N+1, \ldots, T-1} c_{j}\right)^{\left(\frac{2}{1-\nu}+\delta\right) \cdot\left(\frac{2}{1-\nu}+1+\delta\right)^{i-N}} .
$$

Here the second inequality comes from the fact that

$$
\begin{aligned}
\frac{\left(\frac{2}{1-v}+1+\delta\right)^{T-N}}{\left(\frac{2}{1-v}+1+\delta\right)-1}>\frac{\left(\frac{2}{1-v}+1+\delta\right)^{T-N}-1}{\left(\frac{2}{1-v}+1+\delta\right)-1}=\left(\frac{2}{1-v}+1+\delta\right)^{T-N-1} \\
+\left(\frac{2}{1-v}+1+\delta\right)^{T-N-2}+\cdots+1
\end{aligned}
$$



yield

The fact that $\left\{a_{n}\right\}_{n=1}^{\infty}$ is the eventually non-decreasing sequence and inequality (7)

$$
\begin{aligned}
a_{T} & >\left(\prod_{i=N}^{T-1}\left(\max _{j=N, N+1, \ldots, T-1} c_{j}\right)^{\left(\frac{2}{1-\nu}+1+\delta\right)^{i-N}}\right)^{\frac{2}{1-\nu}+\delta} \geq\left(\prod_{i=N}^{T-1} c_{i}^{\left(\frac{2}{1-\nu}+1+\delta\right)^{i-N}}\right)^{\frac{2}{1-\nu}+\delta} \\
& =\left(a_{N}^{N} \prod_{i=N+1}^{T-1} a_{i}\right)^{\frac{2}{1-\nu}+\delta} \geq\left(\prod_{i=1}^{T-1} a_{i}\right)^{\frac{2}{1-\nu}+\delta} .
\end{aligned}
$$

This implies that

$$
a_{T}^{1-v}=\left(a_{T}^{\frac{1+\frac{\delta}{2}(1-v)}{1+\frac{\delta}{2}(1-v)}}\right)^{1-v}=a_{T}^{\frac{1-v}{1+\frac{\delta}{2}(1-v)}} \cdot a_{T}^{\frac{\frac{\delta}{2}(1-v)^{2}}{1+\frac{\delta}{2}(1-v)}}>a_{T}^{\frac{\frac{\delta}{2}\left(1-\frac{1}{2}\right)^{2}}{21(1-v)}} \cdot\left(\prod_{i=1}^{T-1} a_{i}\right)^{2} .
$$

Now we will prove that for every sufficiently large $N$

$$
\sum_{n=N+1}^{\infty} \frac{b_{n}}{a_{n}}<\frac{2 \log _{2}^{2} a_{N+1}}{a_{N+1}^{1-v}}
$$

From (1), (2) and the fact that $\left\{a_{n}\right\}_{n=1}^{\infty}$ is an eventually non-decreasing sequence of positive integers we obtain that

$$
\begin{aligned}
& \sum_{n=N+1}^{\infty} \frac{b_{n}}{a_{n}}<\sum_{n=N+1}^{\infty} \frac{\log _{2}^{\gamma} a_{n}}{a_{n}^{1-\nu}}=\sum_{N<n \leq \log _{2} a_{N+1}} \frac{\log _{2}^{\gamma} a_{n}}{a_{n}^{1-\nu}}+\sum_{\log _{2} a_{N+1}<n} \frac{\log _{2}^{\gamma} a_{n}}{a_{n}^{1-\nu}}<\frac{\log _{2}^{1+\gamma} a_{N+1}}{a_{N+1}^{1-\nu}} \\
& \quad+\sum_{\log _{2} a_{N+1}<n} \frac{\log _{2}^{\gamma} a_{n}}{a_{n}^{1-\nu}}<\frac{\log _{2}^{1+\gamma} a_{N+1}}{a_{N+1}^{1-\nu}}+\sum_{\log _{2} a_{N+1}<n} \frac{n}{2^{n(1-\nu)}} \leq \frac{2 \log _{2}^{1+\gamma} a_{N+1}}{a_{N+1}^{1-v}} .
\end{aligned}
$$

Let $T$ satisfies (6). Inequalities (8) and (9) imply that for every sufficiently large $T$

$$
\begin{aligned}
& \left|\sum_{n=1}^{\infty} \frac{b_{n}}{a_{n}}-\sum_{n=1}^{T-1} \frac{b_{n}}{a_{n}}\right|=\left|\sum_{n=1}^{\infty} \frac{b_{n}}{a_{n}}-\frac{\prod_{n=1}^{T-1} a_{n} \sum_{n=1}^{T-1} \frac{b_{n}}{a_{n}}}{\prod_{n=1}^{T-1} a_{n}}\right|=\left|\sum_{n=T}^{\infty} \frac{b_{n}}{a_{n}}\right| \leq \frac{2 \log _{2}^{1+\gamma} a_{T}}{a_{T}^{1-\nu}} \\
& <\frac{2 \log _{2}^{1+\gamma} a_{T}}{a_{T}^{\frac{\delta}{1+(1-v)^{2}}(1-v)} \cdot\left(\prod_{i=1}^{T-1} a_{i}\right)^{2}}=\frac{2 \log _{2}^{1+\gamma} a_{T}}{\left(a_{T}^{1-\nu}\right)^{\frac{\delta}{1+1}\left(1-\frac{\delta}{2}(1-v)\right.} \cdot\left(\prod_{i=1}^{T-1} a_{i}\right)^{2}}
\end{aligned}
$$

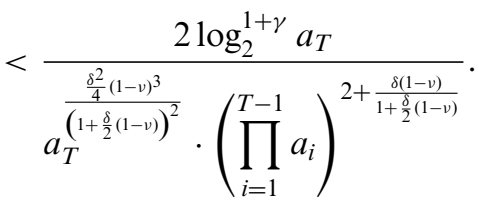


Set $q_{T}=\prod_{n=1}^{T-1} a_{n}, p_{T}=\prod_{n=1}^{T-1} a_{n} \sum_{n=1}^{T-1} \frac{b_{n}}{a_{n}}$ and $\epsilon=\frac{\delta(1-v)}{1+\frac{\delta}{2}(1-v)}$. Because

$$
\frac{2 \log _{2}^{2} a_{T}}{\frac{\frac{\delta^{2}}{4}(1-\nu)^{3}}{\left(1+\frac{\delta}{2}(1-\nu)\right)^{2}}} \text { tends to zero when } T \text { tends to infinity }
$$

we obtain the inequality

$$
\left|\sum_{n=1}^{\infty} \frac{b_{n}}{a_{n}}-\frac{p_{T}}{q_{T}}\right|<\frac{1}{q_{T}^{2+\epsilon}}
$$

which holds true for all sufficiently large $T$.

The fact that we can find infinitely many pairs $\left(p_{T}, q_{T}\right)$ satisfying (10) and the Roth's Theorem [7] imply that the number $\sum_{n=1}^{\infty} \frac{b_{n}}{a_{n}}$ is transcendental.

Acknowledgements. We thank Professor Radhakrishnan Nair from the Department of Mathematical Sciences, University of Liverpool, for his help with the presentation of this article.

\section{REFERENCES}

1. D. Duverney, Transcendence of a fast converging series of rational numbers, Math. Proc. Camb. Phil. Soc. 130 (2) (2001), 193-207.

2. P. Erdös, Some problems and results on the irrationality of the sum of infinite series, J. Math. Sci. 10 (1975), 1-7.

3. J. Hančl, Expression of real numbers with the help of infinite series, Acta Arith. LIX (2) (1991), 97-104.

4. J. Hančl, Two criteria for transcendental sequences, Le Matematiche, LVI (1) (2001), $149-160$.

5. K. Nishioka, Mahler functions and transcendence, Lecture Notes in Mathematics No. 1631 (Springer-Verlag, 1996).

6. A. N. Parshin and I. R. Shafarevich (Eds.), Encyclopaedia of Mathematical Sciences, Volume 44, Number Theory IV (Springer-Verlag, 1998).

7. K. F. Roth, Rational approximations to algebraic numbers, Mathematika 2 (1955), 1-20.

8. A. B. Shidlovskii, Transcendental numbers (Walter de Gruyter, 1989). 